\title{
Correction of Figure
}

In the December 2017 issue of Preventive Nutrition and Food Science (2017;22(4):320-326), a correction is necessary in the article "Kaempferol Inhibits Angiogenesis by Suppressing HIF-1 $\alpha$ and VEGFR2 Activation via ERK/p38 MAPK and PI3K/Akt/mTOR Signaling Pathways in Endothelial Cells" by Gi Dae Kim.

There was an editing error that originated in the publication process. During editing, the same gel figure was presented twice by mistake. The corrected form of Figure 4 is shown below. Also, the final version of the article is available online.

A
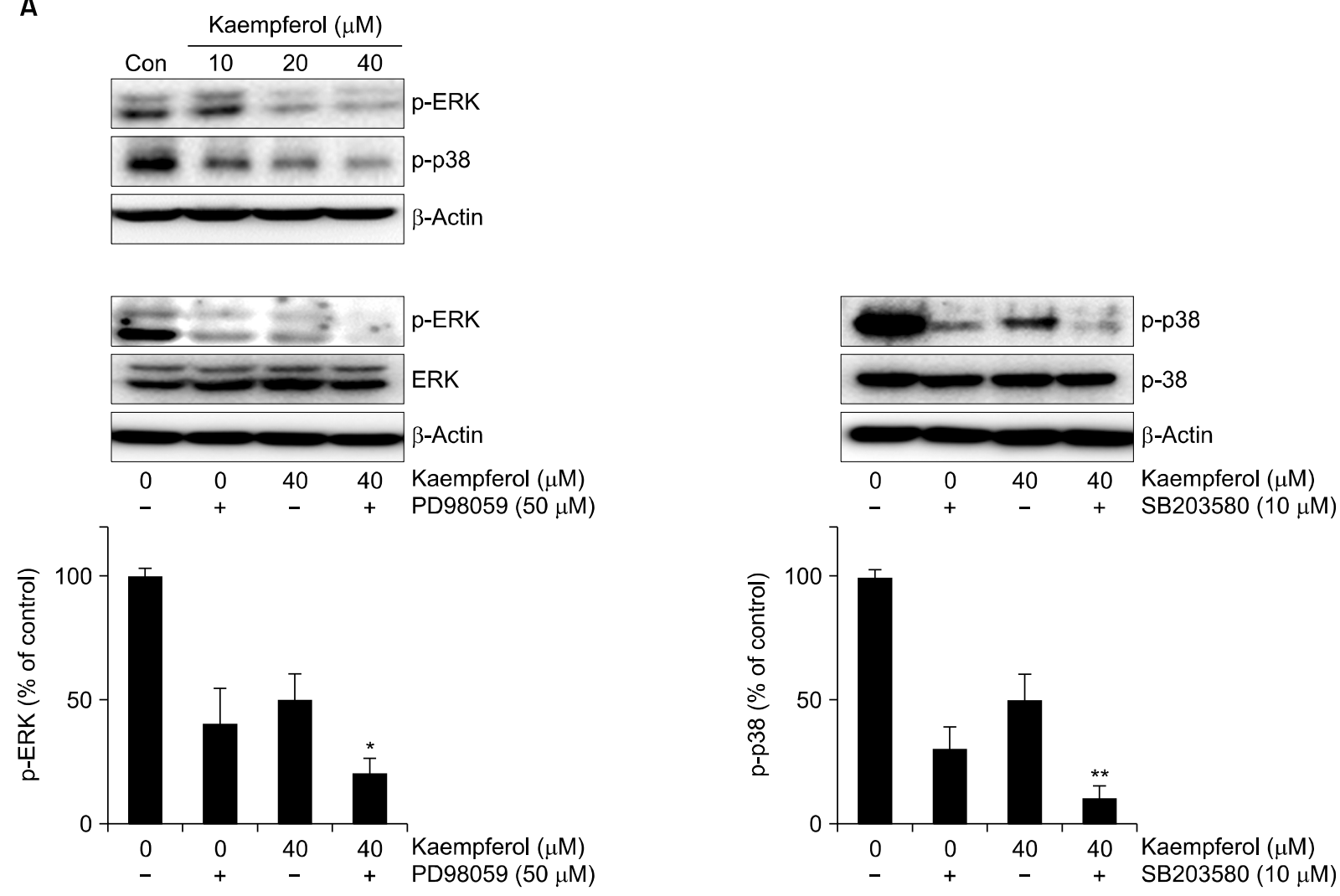

Fig. 4. Effect of kaempferol treatment on (A) MAPK and (B) PI3K/Akt/mTOR signaling pathways. Endothelial cells were treated with the indicated concentrations of kaempferol for $24 \mathrm{~h}$. Protein samples $(40 \mu \mathrm{g})$ were subjected to $10 \%$ SDS-PAGE, and phosphorylated ERK, p38, PI3K, Akt, mTOR, p70S6K, VEGFR2, and HIF-1 $\alpha$ were detected by western blotting. The cells were treated with kaempferol in combination with MAPK inhibitors (ERK inhibitor, PD98059; p38 inhibitor, SB203580), and the activation of ERK and p38 was detected by western blotting; $\beta$-actin was used as an internal control. Values represent the mean \pm SD of three independent experiments. ${ }^{*} P<0.05$ and ${ }^{* *} P<0.01$ compared with control. 
B
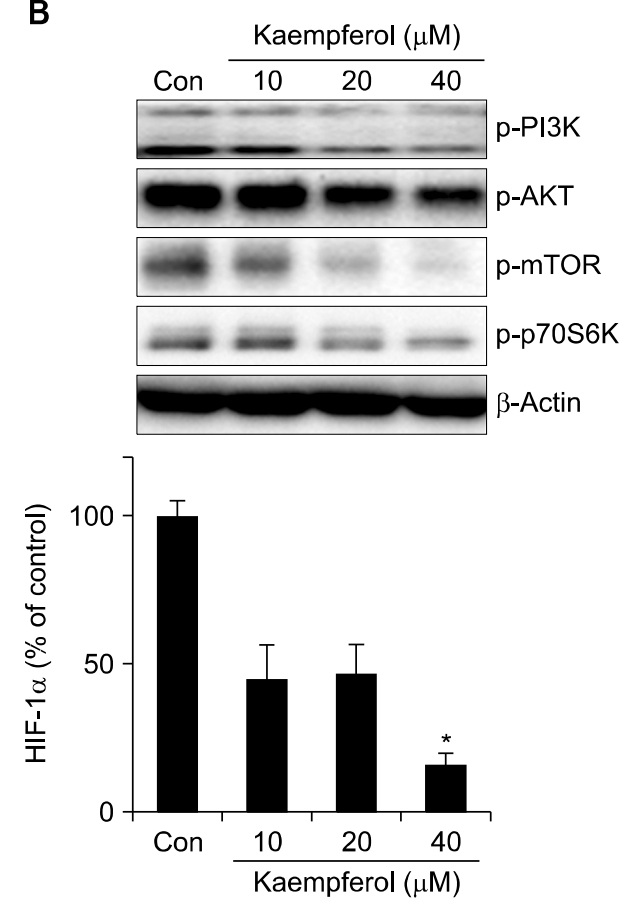

Fig. 4. Continued.

These amendments do not alter the conclusions of the paper.

The editors apologize to the readers for the error.
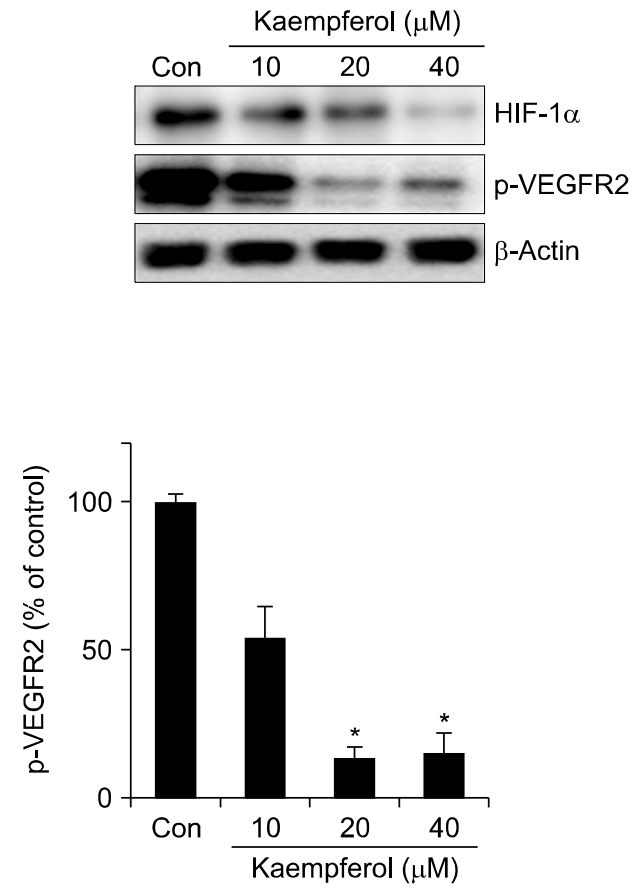